\title{
A WEB-BASED FRAMEWORK FOR URBAN DATA SHARING AND DYNAMIC INTEGRATION
}

\author{
Hongxia Wang, Andy Hamilton, John Counsell, Joseph Tah
}

The Research Institute for Built and Human Environment (BuHu)

http://www.buhu.salford.ac.uk/

University of Salford, Salford, UK

Construction and Property Research Centre,

FBE, University of the West of England, Bristol, UK

H.wang@salford.ac.uk

Remisión Artículo: 30-4-2007

Keywords: Web feature service (WFS), Data integration, Mediator, Building feature service (BFS)

\begin{abstract}
In urban planning and management, data sharing and integration are always problematic because urban datasets are heterogeneous and scattered in different domains and organisations. This paper introduces the research work being carried within the Virtual Environment Planning (VEPS) project. Based on a loosely-coupled mediator system and Open Geospatial Consortium's Web Services (OWS) architecture, a Web-based information service framework is designed to support the sharing and integration of geo-spatial information, building information and other related information. In this framework, a Building Feature Service (BFS) is defined to implement the retrieval of building information model on the Web according to the OWS principles. The BFS can act as a Web-based building information server for urban planning and construction stakeholders. As proof of concept, an initial prototype is developed to demonstrate the data sharing and integration based on this web-based information service framework.
\end{abstract}

\section{Introduction}

In urban planning, there is an increasing need to seamlessly utilise and integrate various urban datasets, described as 'urban data fusion'. The INSPIRE draft directive defines a need for "a coherent combination of spatial data sets or services that represents added value, without requiring specific efforts on the part of a human operator or a machine" (INSPIRE 2004). Significant integration of relevant urban datasets like spatial data from the CAD and GIS domains will help both professionals and the public in their understanding of existing urban environments (Nedovic-Budica 2004; Oosterom 2005; Döllner 2006). Professionals like urban planners, designers, managers and developers need the integrated information in many working tasks like urban planning, urban design, preservation of historical buildings, disaster management etc. Citizens can also benefit from the visualization and simulation based upon integrated information.

However, data sharing and integration have always been problematic in urban planning due to data heterogeneity and its cross-domain and cross-organisation nature. First, the datasets relevant to planning are heterogeneous. Data heterogeneity exists on syntactic, schematic and semantic levels (Bishr 1998a; Visser 2001). Each data source may model the world in its own way and be incompatible with the others. Secondly, these datasets are from different domains and stored in a variety of systems and formats. For example, the spatial data from the CAD and GIS domains are represented by different modelling methods and coordinate systems. Furthermore, these datasets relevant to planning are managed by different organizations and 
distributed in various locations. The owners of such data may be unwilling to share their information openly with others. So it is difficult to locate, access or extract data from other sources in a reliable and acceptable form.

The main aim of this research is to investigate the technical methods with which to support data sharing and integration for urban planning applications. Some pilot research work has been done in the EC funded IntelCities project (FP6 6.7 million euros, 2004-2005). A conceptual building data integration system was implemented that achieved the integration of digitised historic building data with other types of city data (Hamilton 2005; Wang 2005). Based on this work, further research is proceeding within the EU-funded VEPS project (INTERREG IIIB, 2 million euros, 2005-2008) in which the University of Salford and the University of the West of England (UWE) are involved. The University of Salford is responsible for developing the VEPS architecture while UME are leading the requirements annalysis task. In this paper, the main focus is to develop a common information service layer of the VEPS architecture based on the requirements analysis.

This paper presents the design of an information service framework that can support data sharing and integration for urban planning. In the next section, some requirement analysis work within the VEPS project is introduced. In section 3, the related work on information integration and interoperability from different communities is presented. In Section 4, a web-based information service framework is designed to accord with the requirements of urban planning. In section 5, the Building Feature Service (BFS) is defined based on the idea of OGC's Web Feature Service. And finally the development of a prototype is introduced as a concept proof for the information service framework.

\section{VEPS and information requirement for current planning system}

\subsection{VEPS project}

The Virtual Environment Planning (VEPS) project is funded by the INTERREG IIIB North West Europe Programme running from November 2004 to April 2008. The VEPS project has eight academic and industrial partners across the NWE region. It is led by the Environment Agency for England and Wales (UK), with:

The University of Salford (UK);

The University of the West of England, Bristol (UK);

Scientific \& Technical Building Centre (CSTB, France);

Manchester Digital Development Agency (UK);

Clementine Media (UK);

Stuttgart University of Applied Sciences (Germany);

The University of Freiburg (Germany).

The VEPS project aims to improve the knowledge base on the potential of Information and Communications Technologies (ICT) for territorial development in the North West European (NWE) region specifically on the use of 3D visualisation, ICT for e-Planning, consultation and communication of citizens' views on planning issues. The main objective is to demonstrate the use of interactive three-dimensional (3D) Virtual Reality visualisations to present highly complex information in a simple and easy-to-understand way to citizens, while allowing their comments and participation. 


\subsection{Requirement Analysis for VEPS}

Urban planning depends on the availability of reliable data/information from different sources. Suitable tools are needed to integrate this data/information to provide three-dimensional (3D) and two-dimensional (2D) models. In the first stage of VEPS, the main task was to define the user requirements which is led by UWE.

Several workshops in the various VEPS partners' countries have been organised. The workshops include: Tours Workshop (12 May 2006, France); Nijmegen Workshop (17 May 2006, Netherland); Bristol Workshop (19 May 2006, UK); Manchester Workshop (22 May 2006, UK). The investigation includes understanding what information is required and what information interpretation and processing tools are needed.

Also a pilot application "Rosensteinviertel redevelopment" is being carried out. The Rosensteinviertel is an urban area in central Stuttgart, Germany that will undergo extensive changes as part of a large development project - Stuttgart 21. Redevelopment plans include proposals to move several railway lines underground and to build a mixed-use development and an improved Rosenstein Park. These plans will result in large-scale changes to the centre of Stuttgart and the existing transport system, and will affect those living and working in the area. This specific scenario also helps the understanding of the user requirements.

This paper will focus on reporting the result of requirements analysis on data issues.

What types of data are required?

Generally speaking, the investigation from the workshops and the pilot application show that the professionals from planning and architectural fields are interested in very specific data and often need special technical know-how. While citizens groups are interested in generally understandable information, in easy-to-use tools with quick responses (http://www.veps3d.org/).

The common data types required in urban planning include:

-Spatial data: a standard set of geographic data, detailed building models, digital terrain models, LiDAR data etc.

-Thematic data: various themes of urban environment like noise, flooding data etc.

-Administrative data: information about planning regulations, planning process management, public participation etc.

Specifically, in the VEPS pilot application "Rosensteinviertel redevelopment", the data requirements were defined as: GIS and CAD data, meta-data, 3D-data (CityGML, 3D Max, VRML), DGM (digital landscape model), air flows and orthophotos, textures for existing facades etc., statistical data, city model for urban area.

What kinds of data processing are needed?

The investigation also shows that current urban planning is still limited to text or 2D maps without real interactive $3 \mathrm{D}$ visualization. In order to present realistic $3 \mathrm{D}$ city models it is necessary to integrate geospatial data with CAD, LiDAR and other data sources. Furthermore, VEPS will need to consider data about context in addition to its visual model. For example, in order to formulate new development proposals that will gain acceptance, there is a need for detailed analysis of the current context. The challenge for VEPS is how to access and integrate various spatial datasets and relevant datasets to support comprehensive analysis and visualisation. 


\subsection{Issues raised in workshops}

Generally, several issues are raised in the workshop discussions regarding data processing. Tools are needed to resolve these issues.

First, information availability and accessibility issues have been addressed in the workshops. It was asked in the Manchester workshop: "Data availability may determine the success of the system. What data is available within organizations? Will the data made available be accurate? ". Attendees at the Manchester workshop also suggested that "enabling access to all data associated with planning in a 'one-stop-shop' (e.g. local authorities, environment agency, etc.) would be useful. Some land purchases for developers have fallen through due to the time taken to go through the planning system". There was a similar view in the Bristol workshop: "Sharing this type of information is good for everyone. Public authorities have a duty to make this information available".

It is always difficult for urban planning stakeholders to assess the information because the data sources may come from different domains and be distributed in different organisations. Sometimes the information in reality exists, but the fact is not known, or it is not easily accessible, leading to the unnecessary duplication of effort and resources. For the information availability and accessibility issues, tools are needed to support information sharing.

Second, information compatibility and interoperability issues were also addressed. The Bristol workshop raised concerns over integration between local authority departments (e.g. planning and transport): "Compatibility is a concern. There is a variety of 3D packages but little compatibility within the industry as a whole to draw all the relevant expertise together". It was claimed in the Manchester workshop that: "Interoperability is important. Can you see what other major developments are going on in the area to see cumulative effects? There needs to be some commonality".

The datasets required are difficult to integrate due to heterogeneity between different data nodes. For example, integration of CAD data into the geospatial infrastructure is an open issue and a major challenge. Information tools should promote connectivity between data nodes and other information networks through the use of open and accepted standards thus facilitating data integration and reducing inefficiencies; system should not require the use of specific "brands" of hardware or software. For information compatibility and interoperability issues, it is necessary to provide tools to seamlessly integrate the heterogeneous datasets.

In summary, data sharing and integration tools are needed in order to resolve the issues of information availability, accessibility, compatibility and interpretabilities.

\subsection{Data operation requirements in Rosensteinviertel scenario}

Specifically, the VEPS pilot application "Rosensteinviertel Stuttgart" defines the common requirements for data operability:

\section{--Data exchange}

Different systems are used in public authorities and planning departments / offices: CAD, various GIS; different data formats ( $\mathrm{dxf}, \mathrm{dwg}$, gis-files, etc). This is also identified as not only technical problems but also lack of knowledge. This leads to the conclusion that data exchange/interaction has to be an atomized process.

\section{--Data access}

An open GIS interface should be supported for central access, 


\section{--Import and export of data / information}

It is necessary to import and export different kinds of data and to support different forms of presentation and different ways of using the data.

\section{--Administration of data}

It is necessary to provide for data administration of and cross-linking of datasets.

\section{--Size and quality of data transfer capable for all kinds of Internet connections}

It is important to minimise data volumes for accessibility for all potential users while maintaining a consistently high presentation quality.

In summarising the investigation of the workshops and the "Rosensteinviertel Stuttgart" application scenario, the data required for urban planning is always a mixture of spatial, thematic and administrative datasets. It is also very essential to support the data sharing and integration in the development of urban planning support tools for the VEPS project.

\subsection{Related Works}

In order to achieve data sharing and integration for urban planning, it is necessary to understand current research efforts from relevant communities. Here we review the data integration approaches from the database community; the efforts in developing relevant web geospatial data service specification from the international organisation the OGC and the development of Spatial Data Infrastructure (SDI) by the European Commission (EC).

\subsection{Data integration approaches}

A lot of research has been done to integrate heterogeneous data sources in the database community during recent years. There are a number of proven and well-established methods that allow heterogeneous data sources to be integrated (Fileto 2001; Stoimenov 2002). Typical examples include database gateways like ODBC or JDBC, federated database systems (Sheth 1990), data warehouses (Voisard 1999), and mediator systems (Wiederhold 1994).

In general, the major data integration approaches fall into the following two groups (Tatbul 2001; Reinoso-Castillo 2003):

- A tightly coupled approach (for example, data warehousing): This approach is also called the eager approach or the materialized view approach. In this approach, data from heterogeneous distributed information sources is gathered, mapped to a common structure and stored in a central location. An advantage of this approach is that users can manipulate different kinds of data in a single operational framework. However, this tightly coupled approach can not support the autonomy of legacy systems, and it is not easy to ensure that the information in the warehouse reflects the current contents of the individual sources.

- A loosely coupled approach (for example, federated database and mediator system): This approach is also called the lazy or virtual view approach. This approach tries to combine existing operational schemes for different forms of information into a well-known existing scheme. All data sources are kept at their original locations. Data is accessed from the sources on-demand when a user submits a query. The query results are up-to date. The drawback of this approach is the development cost and accessing efficiency. 


\subsection{OGC Web Service (OWS)}

Geospatial information is essential for urban planning applications. Various GISs have been developed to analyse, process, and manage spatial data which are always using proprietary storage formats. The integration or conversion of the spatial data is resource intensive. There have been continuous efforts to integrate multiple sources and multiple formats within geospatial information (Abel 1998; Claramunt, Jiang et al. 2000; Garton 2001; Butenuth 2003). The Open GIS Consortium (OGC) is making particular efforts to overcome these problems and to develop implementation specifications for the interoperability of the spatial data.

The OGC (http://www.opengeospatial.org) is an international organization promoting the interoperability and sharing of geospatial resources and services in the distributed environment through the development of consensus-based implementation specifications. OGC specifications are widely used by geospatial communities for sharing data and resources and some of the specifications are becoming ISO standards. For example, OGC's Geographical Mark-up Language (GML), as a XML-based schema for modelling, transport, and storage of geospatial information, was developed as a data exchange standard.

OGC is developing specifications for standardizing the interfaces of spatial web services. Web services implementing these specifications are called OGC Web Services (OWS). OWS is an evolutionary, standards-based framework that will enable seamless integration of a variety of online geo-processing and location services (OGC 2005). OWS will allow future applications to be assembled from multiple, network-enabled geo-processing and location services. This capability will be possible because rules will be established for these services to advertise the functionality they provide and how to send service requests via open, standard methods. OWSs will allow distributed geo-processing systems to communicate with each other using technologies such as XML and HTTP. OGC Web Services will provide a vendor-neutral interoperable framework for web-based discovery, access, integration, analysis, exploitation and visualization of multiple online geo-data sources, sensor-derived information, and geoprocessing capabilities (Schilcher 2005).

OGC's OWS includes a number of standard specifications:

- Web Map Service (WMS) -portrayal service

- Web Feature Service (WFS) - data service

- Web Coverage Service (WCS) -data service

-Web Terrain Service ( WTS ) -portrayal service

-Web 3D Service (W3DS) -portrayal service

For WMS and WTS, the whole rendering is done on server side and the output map formats are either "picture" formats or "graphic element" formats in client side. W3DS merges different object types into a single scene graph, representing a visual representation of the basic 3D geodata. The semantic characteristics and relations are not contained in a scene graph (OGC 2005). The WFS and WCS are providing the semantic information of features. These standard specifications allow for server requests using an ID, bounding box or specific spatial and other operators. The selection of an appropriate interface for the service is not fully independent of the chosen transfer format. Considering the data sharing and integration goal of this research, the focus will be on the data service specifications like WFS and WCS.

\subsection{Spatial Data Infrastructure (SDI)}

A spatial data infrastructure provides a basis for spatial data discovery, evaluation, download and application for users and providers within all levels of government, the commercial sector, the non-profit sector, academia and the general public (Holland 1999). Spatial information is increasingly becoming the important content of many applications. This is driving forward the development of SDI at national, regional and global levels i.e. National Spatial Data Infrastructure (NSDI) and Global Spatial Data Infrastructure (GSDI). 
INSPIRE (Infrastructure for Spatial Information in Europe) (http://www.ec-gis.org/inspire/) is an initiative launched by the European Commission and developed in collaboration with Member States and accession countries. It aims to make available relevant, harmonised and quality geographic (spatial) information to support formulation, implementation, monitoring and evaluation of Community policies with a territorial dimension or impact. It will address technical standards and protocols, organisational and co-ordination issues, data policy issues including data access and the creation and maintenance of spatial information. INSPIRE will pave the road for a progressive harmonisation of spatial data in the Member States of the EU (INSPIRE, 2004). The INSPIRE directive will follow international standards to facilitate the integration of information so that it can be available for use to provide enhanced public services.

One of the main principles of the INSPIRE program is that "data should be collected and maintained at the level where this can be done most effectively". In this sense, local, regional, national and European spatial datasets will be made accessible independently from their own physical locations.

The philosophy of integrating existing datasets to facilitate more holistic applications is a key motivation for this work. The design of information layer for VEPS should enable public and private agencies to share mapping data and distribute data changes and updates in near realtime based on the INSPIRE principle. This is also consistent with the requirements analysis in the VEPS project.

\subsection{A Web-based information service framework}

The authors are working on a solution to provide information support to advanced urban planning applications. A Web-based information service framework was designed based on the requirements analysis result of the VEPS project and current reseach development. This frameowrk will acts as the information layer in the VEPS common architecture.

\subsection{The design of information service framework}

According to the requirement analysis carried out within the VEPS project, data sharing and integration are regarded as two important issues for data handling in the VEPS architecture.

As discussed above, the available datasets in one test site often come from different sources and they may be distributed in different sectors which are usually unwilling to give their data to others. It is necessary to provide information sharing and integration of heterogeneous datasets from different organisations and give users an integrated view of the diverse datasets. This is essentially a problem of data sharing and dynamic information integration. In order to design an information service framework to provide information support for urban planning, the following aspects need to be taken into considerations:

- Data standardisation: Datasets are from different sources and are in various data structures and schemas. In resource transformation level, interoperability needs to resolve heterogeneities of various datasets. There are many significant advances in data standardisation (Murray 2002; Wang 2005). The design of this information service framework should align with these widely accepted standards as much possible. The OGC's GML is a big contribution to this level.

- Integration methods: Integration the data sources and get the comprehensive information can greatly facilitate the urban planning decision making process. In the traditional way, for one specific application, all required information will be collected and physically combined together. This one-off method always incurs of high cost of acquiring and integrating the various datasets. And these kinds of combination will lose its value once the specific application finished. It is often the case that some data vendors or owners provide basic geo- 
information while others provide thematic information (Zhang 2000). A generic solution should employ loosely-coupled integration system to let user logically refer to the required data in distributed environment and allow the dynamic on-fly combination.

- Data discovery mechanism: Datasets are owned by different organisations. Sometime the planning stakeholders even don't know what data sources are available. It is difficult for the stakeholders to find the right information for the right application. These data sources should have their metadata descriptions and catalogue services which provides methods of data discovery.

- Distributed computing architecture: Implementing the information service in a distributed environment relies on technical architecture adopted. Distributed computing can resolve interoperability of platforms and provide the information exchange mechanism. Distributed computing technologies such as CORBA, DCOM and Web service are candidates for providing distributed services. The OWS provides the definition of delivering spatial and thematic data over the Internet using HTTP protocol.

According to these considerations and current technical developments as reviewed in last section, two main technolgies: mediator integration systems and the OGC compatible web services, are employed to support the sharing and integration of distributed spatial data sources for urban planning. The design of this Web-based Information Service Framework by the principal author is shown in Figure 1. Such an open and flexible architecture will find its main uses in situations very similar to the SDIs. But this framework is specifically for urban environment with smaller scale and richer details. This framework uses GML profile(XMLbased) to model and transport information. It mainly comprises four layers: data source layer, wrapper layer, mediator layer and application /client layer.

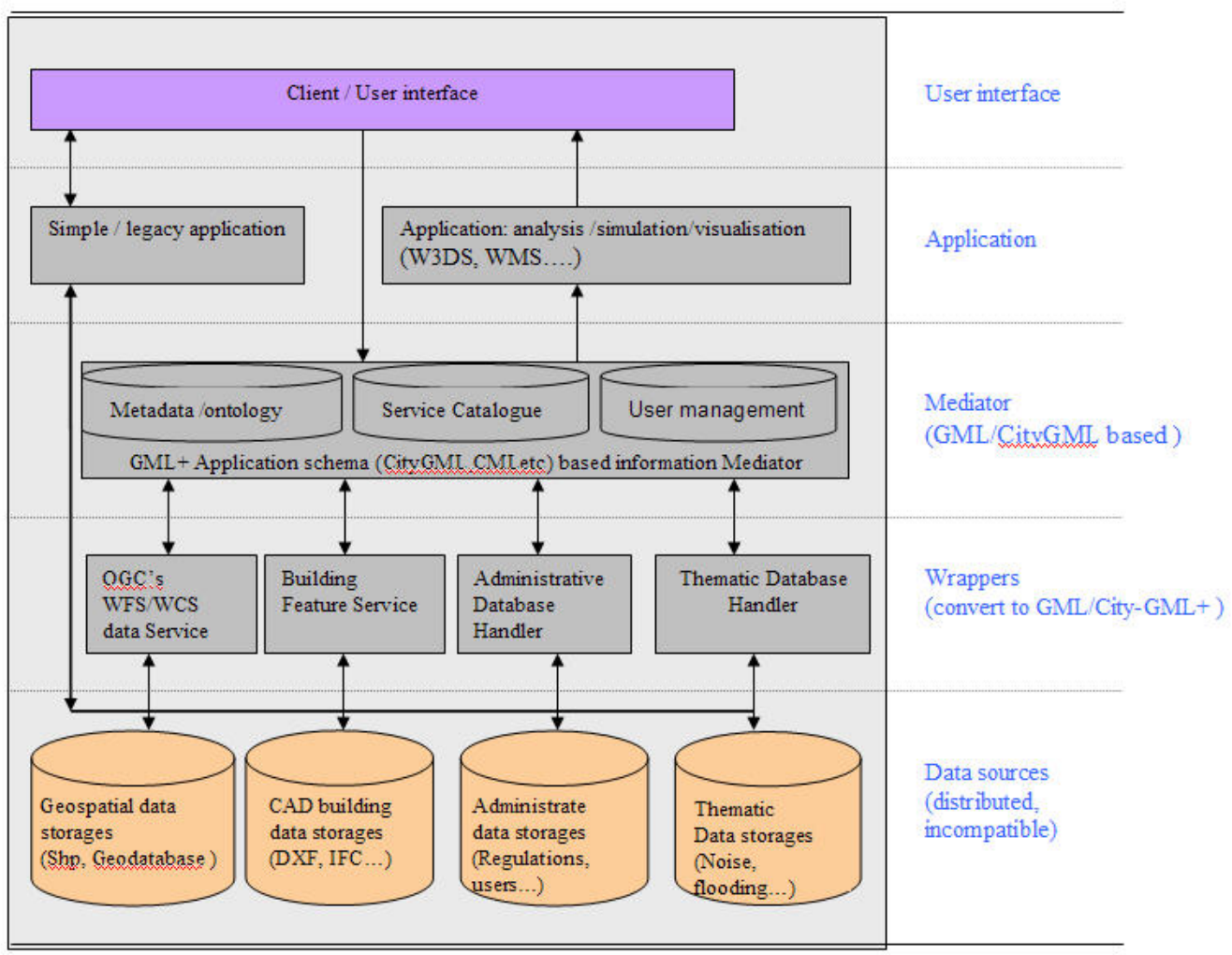

Figure 1: Information service framework for urban planning 


\subsection{Common data model}

Within this framework, data source heterogeneity is managed by wrappers which export a common view for each data source.

A common data model can be seen as a virtual view over the existing internal models of data sources. This common data model is essential to enable transformations of the inputs and outputs of different models so that models can be combined. In order to have a realistic level of data integration and data sharing, the common data model must be used as a basis for describing the information to be shared within urban planning.

The design of a holistic common data model for urban planning is a difficult task. The common data model must cover the definitions of urban planning required data. A series of data standards and specifications are increasingly developed and used worldwide. The common data model in this information service framework is based on OGC's GML and GML application schema. The reasons for using GML and GML application schema as the common data model for information exchange are as follows:

- $\mathrm{GML}$ is XML-based which ensures platform independence and easy implementation.

- GML is popullarly supported by many vendors as well as national and international organisations.

- GML application schema is flexiable and extensiable. The design of application schema can be carried out in different domains. OGC have provided the specification for design of GML applcation schema.

- Data providers can be any systems with any data formats as long as they can map their datasets into XML documents.

- It is easy and efficient to transfer data in the XML format in distributed web environemnt.

- The output XML document can be used in any applications as long as the applications can parse XML documents.

In the prototype, CityGML, as a GML application schema, is employed as the basic common data model. CityGML has been published as a candidate OpenGIS implementation specification for discussion by OGC. It is fairly easy to extend CityGML by adding other GML application schema to make it into a real 'common' data model. In Section 6, there will be more introduction about CityGML.

\subsection{Data source layer}

In VEPS, there are three kinds of datasets: spatial data, thematic data and administrative data. They are included in the data source layer.

\section{-Spatial data}

Spatial data is the core necessity for 3D City Models. According to the VEPS specification, the basic spatial data is a standard set of geographic data that is common to all test sites. In particular, a CAD building model is needed for $3 \mathrm{D}$ visualisation since buildings are the most important element in the urban environment. The main spatial data can come from either the GIS or CAD domain. The related common standards are respectively GML for geospatial information and Industry Foundation Classes (IFC) for building information modelling (IAI 2002).

\section{-Thematic data}

Thematic data includes various themes within the urban environment such as noise, flooding data etc. These datasets can be used to implement simulations. These datasets might differ between test sites. Different themes may have their domain standards. The thematic datasets and simulation results can be geo-referenced. 


\section{-Administrative data}

These datasets can help the local government to manage the planning process, such as information about planning regulations, public participation, administration etc. For example, how to describe the participants' comments information on a planning proposal and make it geo-related is investigated and described in the VEPS project.

\subsection{Data wrappers}

A wrapper is a piece of software which is developed to extract data from a data store and provide the required data to the mediator layer in a common data model (Wiederhold 1992). The wrappers encapsulate details of each data source, allowing data access using a common data model. One wrapper has to be developed for every different data source. Wrappers should be managed by data owners considering the close link with the data sources. Data owners can decide what kinds of data can be accessed by different users.

For geospatial data, the OGC's data service can be employed as a wrapper. The burden of developing wrappers is greatly reduced since the OGC's Web Services are strongly supported by many commercial and open source GIS software packages. Web Feature Service (WFS) allows a client to retrieve and update geospatial data encoded in Geography Markup Language (GML) from multiple Web Feature Services. A server that implements the OGC WFS specification can distribute geographic features to a client application. WFS can be invoked by submitting requests in the form of URLs (GET/POST requests) (OGC 2005a).

For CAD information, currently there is no similar facility to match the OGC's WFS. The principal author defined the concept of Building feature service (BFS) which can act was a wrapper for building information model. This is based on the idea of OWS's data service. The BFS can retrieve building features from CAD building models encoded in the common data model CityGML. The detail description of the BFS will be given in the following section.

The other datasets describe the specific adminstrative information of urban planning or cover different themes like air pollution, noise, flooding. The development of these wrappers relies on the knowledge of domain experts and developer. For example, the wrappers of planning policy and regulation need to extract the planning criteria from a planning regulation document which must be based on planners' knowledge. The information will help the development of proposals as well as the consultation process by keeping options within the realm of what is permissible and practical.

\subsection{Mediator layer}

A mediator is a software module that exploits encoded knowledge about some datssets to create information for a higher layer of applications (Wiederhold 1992). The mediator layer offers an integrated view of the data supplied by a collection of sources. The integrated view is supported by a common data model.

The mediator has the description of data sources in metadata/ontology repository. Also the mediator has the catalogue of data wrappers. It also manages information like user authentication. The descriptive and management information can reflect the needs of its potential users. But it does not maintain a database of objects.

The mediator dispatches user queries to the distributed heterogeneous data sources and assembles query results. First, the mediator can receive a request from a client. Based on metadata and service catalogue, the request is decomposed into sub-requests to the wrappers of distributed heterogeneous data sources. The wrappers retrieve the requested information in the common data model and send the query results back to the mediator. Then the mediator assembles the query results and replies to the client with the combined result. 
Since we employed GML application schema as the common data model which is XML-based, the assembly work is relatively easy which mainly includes the merging of XML documents. However, the despatch of a query is always a challenge for the development of a mediator.

\subsection{BFS (Building Feature Service)}

In VEPS, 3D urban environment visulisation should include detailed building information based on the requirement analysis results. Buildings are the most important elements in urban environment. Most urban planning tasks are closely related to buildings. In this information service framework, Building Feature Service (BFS) is defined to retrieve building information and acts as a wrapper of Building Information Model (BIM). The design of BFS is based on OGC's WFS.

\subsection{From WFS to BFS}

The OGC's Web Feature Services (WFS) interface specification is to provide a reputable structure and mechanism for the query and retrieval of geographic features (Reed 2005). Users can obtain requested objects at feature level based on their specific working contexts. The data presentation and rendering are not controlled at server level. This provides some flexibility for users to decide the display contents.

However, WFS is designed for geo-spatial information. In most of current geo-information sources, the description of building features mainly includes 2D building footprints (with building heights sometimes). Geo-information based visualisation is limited to $2.5 \mathrm{D}$ which is not particularly impressive for either professionals or public. Currently many realistic 3D city models have been developed by integrating CAD, GIS and other data sources together (Jepson, Liggett et al. 2001; Yao, Tawfik et al. 2003). These city model methods focused on rendering and visualisation, little attention has been paid on the semantic information presentation and users can not get the feature level information (Zlatanova et al 2002).

Historically, CAD and GIS have been developed as solutions to different problems in different domains - the former optimized for the modelling of new but well-defined objects; the latter for the reconstruction of existing objects about which only spare and incomplete information is available (Oosterom 2005). The CAD building model has different geometric and semantic definitions from building feature in GIS domain. The OGC WFS's operations for retrieving and manipulating geographic features can not be fully matched for CAD model handling. Therefore, Building Feature Services (BFS) is suggested by the authors to retrieve and manipulate building objects. BFS can then provide building objects information to users according to the similar idea of WFS.

The BFS can directly access building information sources which can be IFC/IFCXML files, OO building model database etc. BFS allows a client to query and update building features on the Web. The exchange documents between client and the Web-based BFS server is XML documents which have detailed building and element descriptions. This detailed building feature description chould be a GML application schema. Currently, the most detailed building model with thematic concept is the building model in CityGML to our best knowleadge. The representation of thematic and spatial aspects of buildings, building parts and installations have four levels of detail from LOD1 to LOD4 (Kolbe T. H. 2005; Gröger 2006).

\subsection{Implementation specification of BFS}

At present, OGC Web Services only support Internet hosting the implementation of the Hypertext Transfer Protocol (HTTP) as distributed computing platform (DCP). An HTTP Uniform Resource Locator (URL) is used to locate the online resource of each operation supported by a service instance. To implement BFS, the first task is to encode user requests in the form of 
URLS (HTPP GET/POST requests). This process is the same as OGC's WFS Keyword-value pairs (KVP) encoding. Keyword-value pairs means that parameters consist of name-value pairs in the form of "name=value" and the pairs are separated by the "\&" character. The details can refer to the OGC's specification (OGC 2005a).

The implementation of BFS is similar to OGC's WFS. Several basic BFS operations are defined as:

-GetFeature: to service a request, and to retrieve feature instances like whole building or building elements such as windows, doors.

-GetCapabilities: to describe its capabilities i.e. service metadata including which building features it can service and what operations are supported on each building features.

-DescribeFeatureType: to request the schema description which describes the structure of any building features it can serve.

For BFS, the common parameters for all operations are URL prefix of web feature, service name (BFS) and request version (Currently 0.3). Some parameter definitions have been changed to suit for the building information model. And the filter definition is modified to set constrains of user requests for building information model.

\section{GetFeature operation}

The GetFeature operation allows to service a request and to retrieve feature instances like whole building or building component parts such as windows, doors from a Building Feature Service (BFS). A GetFeature request is processed by a BFS and an XML document, containing the result set, is returned to the client.

This operation is similar to the WFS's GetFeature specification. Because of the different natures of CAD model and GIS model, some parameters in WFS needed to be redesigned in BFS. For example, the bounding box parameters (BBox) of WFS are not suitable for building models. Instead, we use the parameters, BLOD (Building Level Of Detail). Based on WFS definition and the characters of building information model, the GetFeature specification is defined as in Table 1.

One challenge with implementing this operation is to be able to create and parse XML documents that contain Filters. Filters are used to define constraints on a query. GetFeature requests contain $<$ Query $>$ element which in turn may contain $<$ Filter $>$ element to constraint the query. If no <Filter> element is contained in <Query> then the query is unconstrained and all feature instances should be retrieved.

Table 1- GetFeature specification

\begin{tabular}{|l|l|l|}
\hline URL Component & $\begin{array}{l}\text { Optoinal } I \\
\text { Mandator } \\
\text { y }\end{array}$ & Description \\
\hline $\begin{array}{l}\text { REQUEST } \\
\text { =GetFeature }\end{array}$ & $\mathrm{M}$ & The name of the BFS request. \\
\hline PROPERTYNAME & $\mathrm{O}$ & $\begin{array}{l}\text { A list of properties may be specified for each feature type that is } \\
\text { being queried. Comma is used to separate the properties. A "*" } \\
\text { character or the absence of a value indicates that all properties } \\
\text { should be retrieved. }\end{array}$ \\
\hline MAXFEATURES=N & $\mathrm{O}$ & $\begin{array}{l}\text { A positive integer indicating the maximum number of features } \\
\text { that the BFS should return in response to a query. If no value is } \\
\text { specified then all result instances should be presented. }\end{array}$ \\
\hline
\end{tabular}




\begin{tabular}{|c|c|c|}
\hline $\begin{array}{l}\text { RESULTTYPE } \\
=[\text { results } / \text { hits }]\end{array}$ & 0 & $\begin{array}{l}\text { The resulttype parameter is used to indicate whether a BFS } \\
\text { should generate a complete response document or an empty } \\
\text { response document indicating only the number of features that } \\
\text { the query would return. A value of results indicates that a full } \\
\text { response should be generated. A value of hits indicates that } \\
\text { only a count of the number of features should be returned. }\end{array}$ \\
\hline TYPENAME & $M$ & $\begin{array}{l}\text { A comma separated list of feature types to query. If no value is } \\
\text { specified that is to be interpreted as all feature types. }\end{array}$ \\
\hline $\begin{array}{l}\text { FILTER } \\
\text { (Prerequisite: } \\
\text { TYPENAME) }\end{array}$ & 0 & $\begin{array}{l}\text { A filter specification describes a set of features to operate upon. } \\
\text { The filter is defined as specified in the Filter Encoding } \\
\text { Specification. If the FILTER parameter is used, one filter must } \\
\text { be specified for each feature type listed in the TYPENAME } \\
\text { parameter. Individual filters encoded in the FILTER parameter } \\
\text { are enclosed in parentheses "(" and ")". }\end{array}$ \\
\hline BLOD & $M$ & $\begin{array}{l}\text { BLOD is a positive integer which decribes the Level of } \\
\text { details(LOD) for building features. The LOD specification can } \\
\text { refer to the CityGML which is used in our prototype. BFS } \\
\text { mainly serves the LOD3 and LoD4 building information requests } \\
\text { from CAD building model, since LoD1 and LoD } 2 \text { building } \\
\text { information requests can be served from WFS by assessing } \\
\text { geospatial data. }\end{array}$ \\
\hline
\end{tabular}

As defined in filter specification (OGC 2005b), a filter is used to define a set of feature instances that are to be operated upon. Filters can be used to specify both spatial and/or non-spatial constraints in a query. A filter may include an operation element including spatial, comparison and logic operations. Logical operators may be used to combine spatial operators and comparison operators in one filter expression.

\section{DescirbeFeatureType operation}

The function of the DescribeFeatureType operation is to generate a schema description of feature types serviced by a BFS implementation. The schema descriptions define how a BFS implementation expects feature instances to be encoded on input and how feature instances will be generated on output. The DescribeFeatureType specification is shown in Table 2.

Table 2 - DescribeFeatureType specification

\begin{tabular}{|l|l|l|}
\hline URL Component & $\begin{array}{l}\text { Optoinal I } \\
\text { Mandatory }\end{array}$ & Description \\
\hline $\begin{array}{l}\text { REQUEST }= \\
\text { DescribeFeatureType }\end{array}$ & M & Name of request. \\
\hline TYPENAME & O & $\begin{array}{l}\text { A comma separated list of feature types to } \\
\text { describe. If no value is specified that is to be } \\
\text { interpreted as all feature types. }\end{array}$ \\
\hline OUTPUTFORMAT & O & $\begin{array}{l}\text { The output format to use to describe the } \\
\text { feature. XMLSCHEMA must be supported } \\
\text { (default). Other output formats, such as DTD } \\
\text { are possible. }\end{array}$ \\
\hline
\end{tabular}




\section{GetCapabilities operation}

The GetCapabilities operation is used to request a capabilities document from a building feature service. BFS must have the ability to describe its capabilities by returning service metadata in response to a GetCapabilities request. Specifically, every building feature service must support the Key-Value pair encoded form of the GetCapabilities request over HTTP so that a client can always know how to obtain a capabilities document. The GetCapabilities specification is shown in Table 3.

Table 3 - GetCapabilities specification

\begin{tabular}{|l|l|l|}
\hline URL Component & $\begin{array}{l}\text { Optoinal I } \\
\text { Mandatory }\end{array}$ & Description \\
\hline REQUEST=GetCapabilities & M & Name of request. \\
\hline
\end{tabular}

\subsection{BFS discussion}

As discussed above, the BFS can work as a Web-based building information server from which users can retrieve building objects and elements information.

There are two issues that need to be addressed here. One is the data source of the BFS. Another issue is the BFS's valuation both in urban and construction domains.

Since the information retrieved by BFS is not only geometrical information but also semantic information about buildings, the data sources have to be rich-information building model. The research efforts in the construction domain have led to the building model development from 2D drawing, 3D model into a 4D/nD building information model (Lee, 2003; Aouad, 2006).

In the construction domain, Building Information Modelling (BIM) is a new approach to describe and display the information required for the design, construction and operation of constructed facilities.

BIM can integrate all the relevant aspects into a coherent organization of data that computer applications can access, modify and/or add to, if authorized to do so (NIBS 2005). Currently the most popularly model used by BIM is IFC (Teo Ai Lin 2006). IFC is an open standard data model where building information can be represented and shared between various processes and professionals (IAI 2002). At present, the possible formats of BFS data sources could be IFC/IFCXML files, OO building databases etc.

BFS is defined as a web-based building information service which could be beneficial for both urban and construction management. On one hand, the BFS can convert building information model from the construction domain into the large scale urban environment. It can act as bridge between the construction and urban domains. The urban management can get more detailed and meaningful information of building. On the other hand, the BFS can also provide building and construction information access and sharing among the different actors in the construction domain.

Actually, the geospatial world has seen a transition from file-based approach to database management system (DBMS) approaches and, more recently, service oriented architectures (SOA) in particular OGC Web Services. In contrast, "to date, CAD systems are still dominated by a file-based use, despite the fact that all modern CAD systems have connections to a DBMS" 
(Oosterom 2005). The BFS can be seen as a good attempt to this direction. BFS can make the building information in one stage of building life cycle useful for next stage on the Web.

\subsection{Prototype implementation}

The principal author has implemented an initial prototype based on the above design of information service framework for the VEPS project. This prototype demonstrates the use of this information service framework.

\subsection{The architecture of prototype}

In order to validate the data sharing and integration capabilities of this information service framework, the principal author implemented a prototype which covers all the vertical layers from data source, wrapper, mediator and client. Two typical kinds of data sources were tested -- IFC document and geo-database. They can be distributed in different computers or locations. The CityGML was chosen as the common data model since it is an GML application schema with detailed building model. The BFS was developed and acts as the wrapper of IFC document; Geo-server's WFS implementation acts as the wrapper of geo-information. The mediator was developed to merge the query results (two XML documents) from two wrappers.

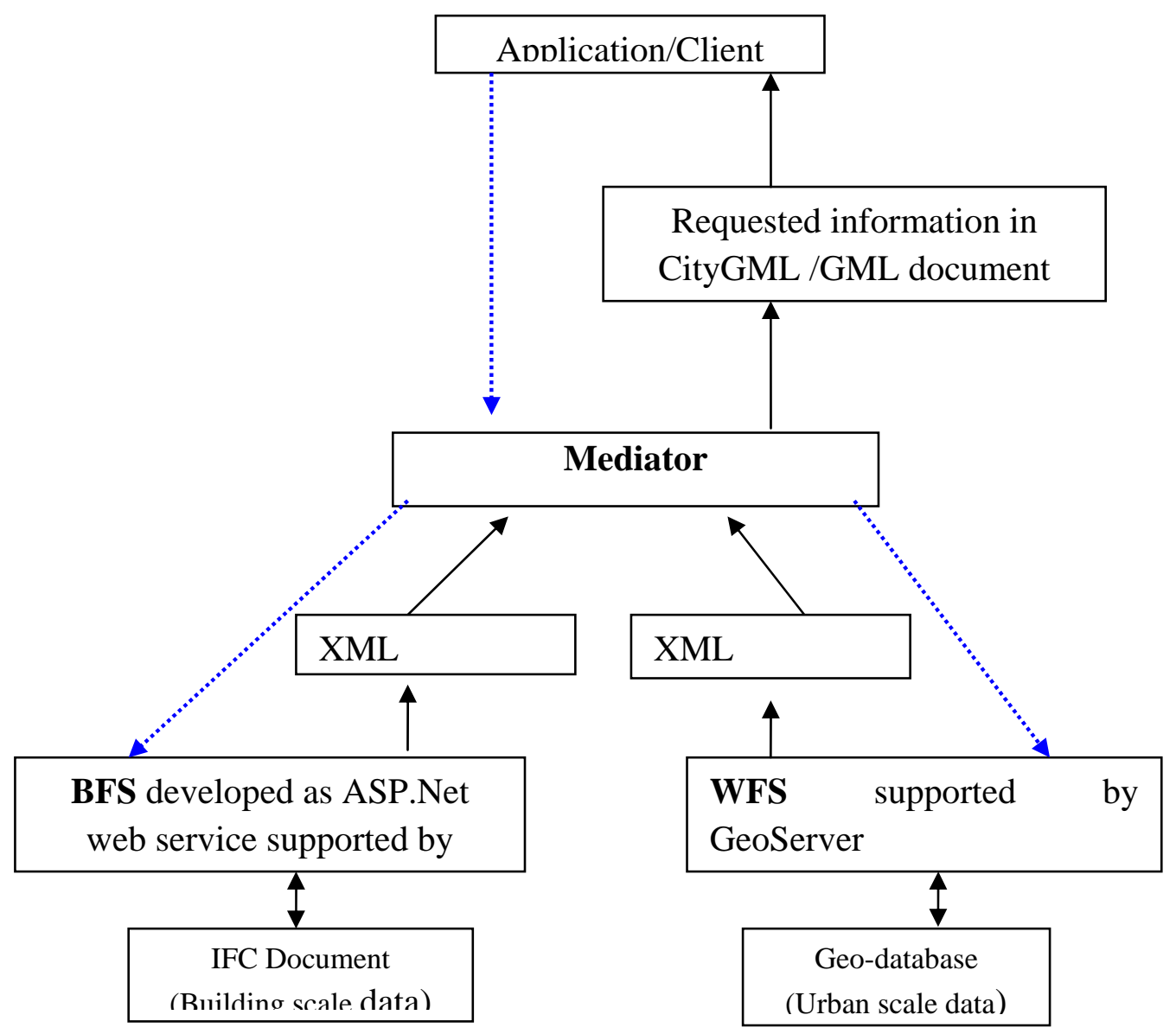

Figure 2: Shows the current implement structure of prototypes as a proof of concept. 


\subsection{CityGML as the common data model}

As stated in last section, GML application schema is employed as the common data model in the information service framework. Using GML standard instead of proprietary data structures as data exchange format can enhance the spatial data sharing and interoperability between applications.

CityGML(http://www.citygml.org/) was originally developed within the scope of the "Special Interest Group SIG3D" from North-Rhine Westphalia (NRW), Germany. The data model is an application scheme based on OGC's GML 3.1. It not only holds geospatial information but also supports a semantic model of city objects. According to (Kolbe T. H. 2005), CityGML is a geometric-topological model which surpport recursive aggregation of objects and geometries; surfaces textured; and thematic attributes assignment to features. CityGML provides support for five Levels of Detail (LoD0 - 4) with different semantic levels of detail (Gröger 2006):

LoD0: a two and a half dimensional Digital Terrain Model (DTM), over which an aerial image or a map may be draped.

LoD1: block models, without any roof structures or textures.

LoD2: buildings have differentiated roof structures and textures. Vegetation objects may also be represented.

LoD3: detailed architectural models with wall and roof structures, balconies, bays and projections. High-resolution textures can be mapped onto these structures. In addition, detailed vegetation and transportation objects are components of a LoD3 model.

LoD4: "walkable" architecture model with LoD3 modelling features plus interior structures like rooms, interior doors, stairs, and furniture.

Since GML was designed by the OGC to serve as the standard exchange format for spatial data infrastructures, processing of CityGML is immediately supported by corresponding web services like the Web Feature Service (WFS) (Kolbe 2005). CityGML is currently the best fitting transfer format for the introduced server requirements on availability of semantic information about building elements and building installations and visualisation (Mas 2006).

Currently CityGML has been published as a Candidate OpenGIS Implementation Specification for discussion by OGC (http://www.opengeospatial.org/standards/dp).

However, CtiyGML is only envisaged as a standard 3D city model with rich semantic information. Some specific data models for urban planning are still under development. For example, the data model of local planning regulations has to be designed in order to develop the capacity to identify what is permissible under local planning regulations. It is fairly easy to extend CityGML by adding other GML application schema to make it into a real 'common' data model.

\subsection{The data sources and wrappers}

In our prototype, there are two data sources with two types of wrappers.

The geospatial data and wrapper

The test geospatial data is Ordnance Survey's Land-Line data that covers the Manchester area. This Land-Line provides a detailed representation of the real world in its plan position (http://www.ordnancesurvey.co.uk/oswebsite/products/landline/). The built environment is in clear, concise detail. There are thirty-seven feature codes, including buildings, roads and their centre lines, water features and tide lines, names and descriptions, administrative and voting boundaries. The scale is the 1:1250 - for urban areas. Land-Line has been withdrawn by Ordnance Survey in 2005. The new realsed Ordnance Survey's data is GML-based MasterMap. However, this does not affect its use as test data sources in our demonstration. 
The wrapper of the geo-information in this framework should be one OGC's WFS implementation. WFS allows uniform direct access to the geo-features stored on a server. A full WFS implementation can perform actions such as:

- query a dataset and retrieve the features

- find the feature definition (feature's property names and types)

- add features to dataset

- delete feature from a dataset

- update feature in a dataset

- lock features to prevent modification

Currently there are several WFS implements available from both commercial vendors and open sources. GeoServer (http://docs.codehaus.org/display/GEOS/Home), an open source GIS software package, is chosen and set up to provide WFS and works as the wrapper of geospatial data in our prototype.

By using the WFS, a small area of geospatial data around Jactin house in east Manchester is requested by sending a request with bounding box parameters.

A GML document is created by the WFS and returned to the client as shown in Figure 3.

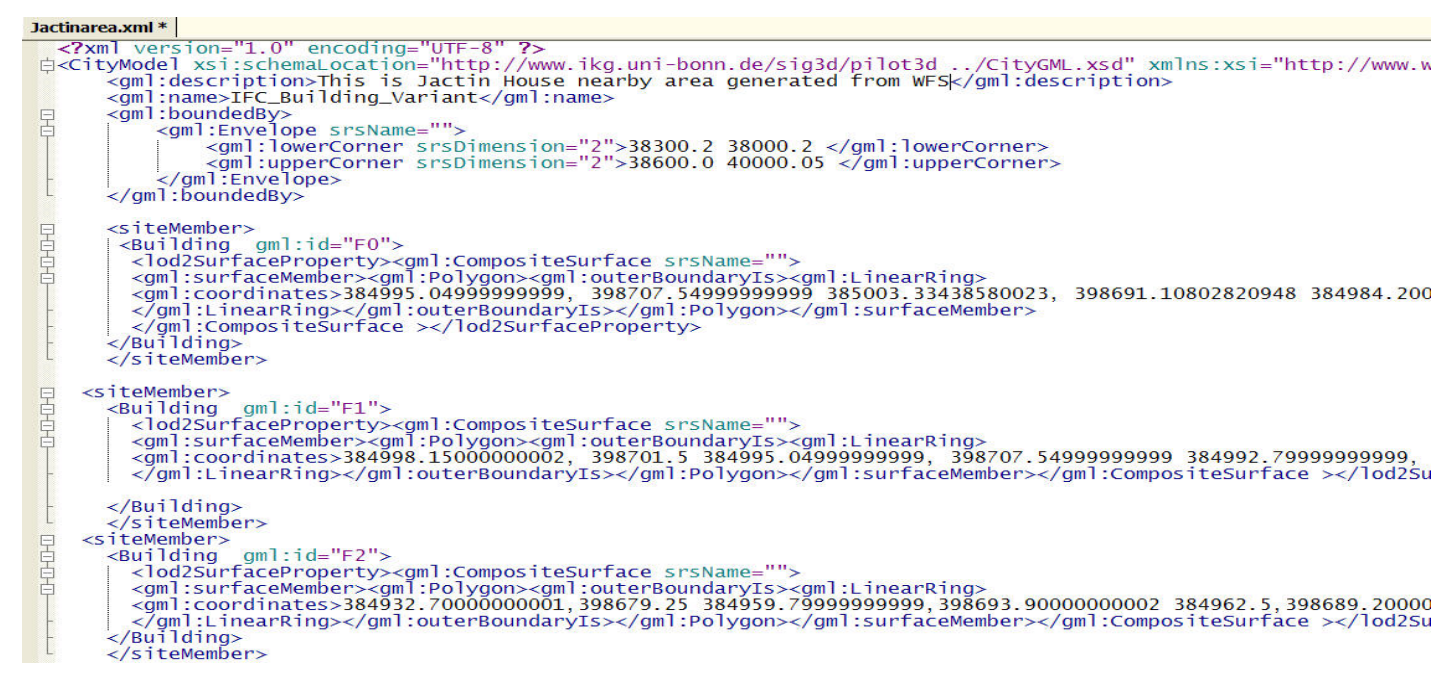

Figure 3: The GML output form WFS of Geo-Server

The IFC building model and wrapper

The test building is Jactin House in Ancoat area of Manchester. The Jactin House data was first captured by using our 3D laser scanner; the raw data were processed into IFC building model by employing some CAD tools (Arayici 2005).

A BFS implementation can act as the wrapper of IFC building model. The BFS was implemented as ASP.Net web service by using C\# programming language based on the Microsoft .Net framework. In the implementation of the BFS prototype, the principal author mainly works on the standard IFC building model because IFC is developed by the International Alliance for Interoperability (IAI) to provide a foundation for the exchange and sharing of information directly between software applications and define a shared building project model (IAI 2002). The BFS's process of generating a CityGML document from IFC building model is 
shown in Figure 4. The main tasks include manipulating IFC/IFCXML document, coordination conversion and generating service's output in CityGML/GML.

For manipulating IFC documents, there are some toolkits like IFCsvr, EDM, Eurostep etc. Also, SAX (Simple API for XML) and DOM (Document Object Model), as two commonly used APIs, can be used for manipulating an IFCXML document. In our prototype, the IFC/IFCXML document manipulation is implemented by using a freeware IFCsvr ActiveX component (http://cic.vtt.fi/projects/ifcsvr/ifcsvrr200/default.html). The main reason we choose is because it is a freeware and easy to embed in the development as an ActiveX components. The shortcoming is that ActiveX components can only be used in Microsoft's platforms.

IFC buildings models are designed in single underlying Cartesian coordinate system called World Coordinate System (WCS). Often a CAD building model has many local coordinate systems where the building elements are defined. The second task of BFS is to transform the required building element into the WCS. The transformation matrix can be calculated based on the relationship between these building elements, specifically in IfcLocalPlacement's PlacementReITo and RelativePlacement attributes in the IFC document.

An XML document is then generated based on the CityGML schema definition. The generation of XML is fairly easy since The System.Xml namespace in Microsoft .Net framework provides standards-based support for processing XML.

In the prototype, the BFS created a CityGML document with LOD3 building description from IFC model of Jactin house as shown in Figure 5.

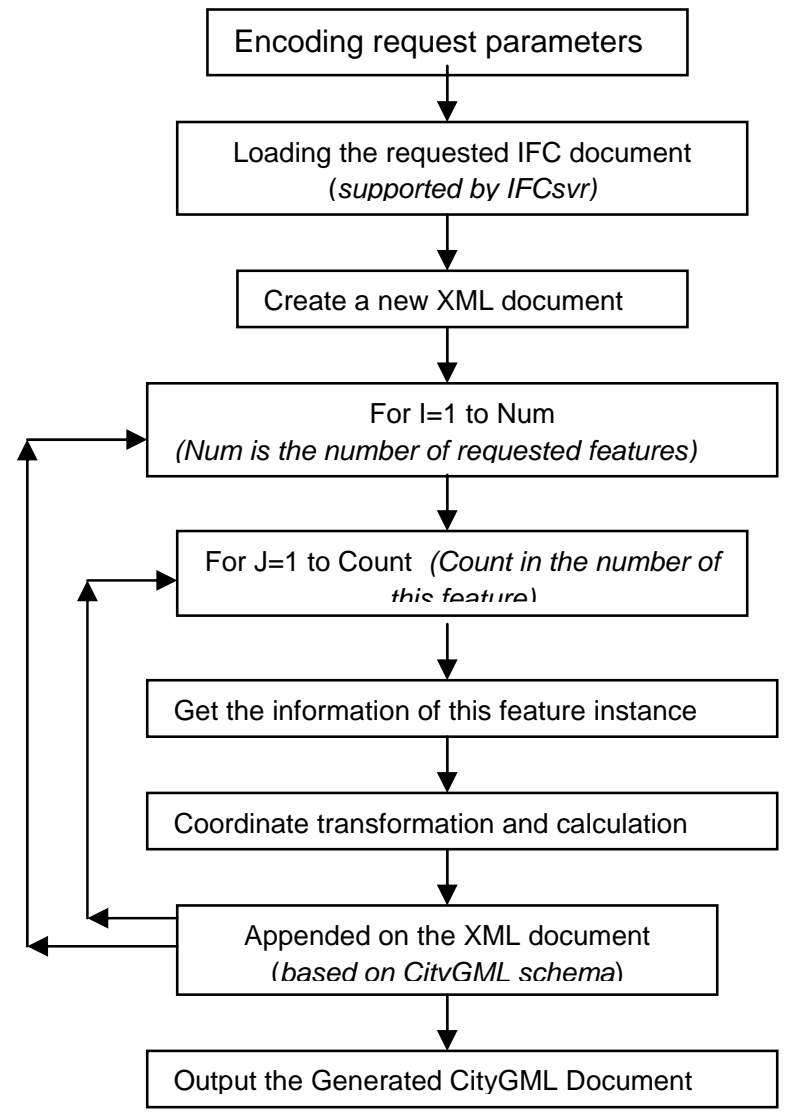

Figure 4: BFS's process of generating a CityGML document from IFC building model 


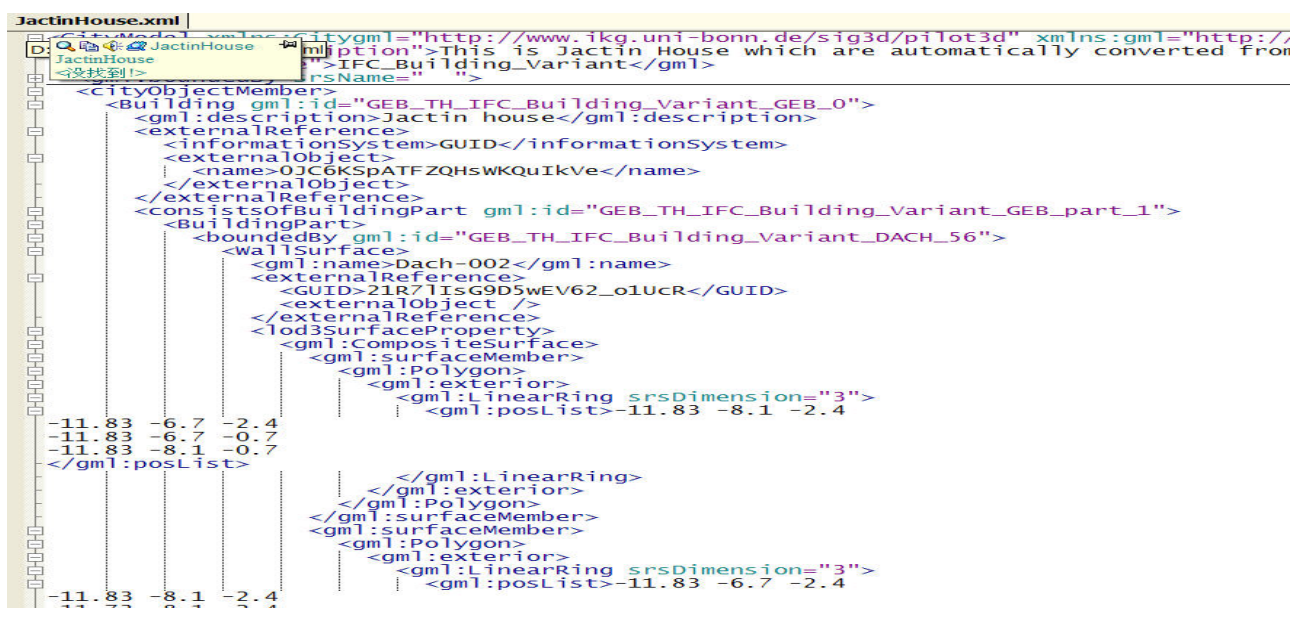

Figure 5. The GML output from BFS implemented by ASP.Net Web service.

\subsection{Development of Mediator}

The mediator is implemented as ASP.Net web service based on the Microsoft .Net framework by using C\# language.

Currently the mediator can support the merging of two XML documents to build a new XML document. Based on the two query results from WFS and BFS, the mediator extracts elements of each XML document and puts them into a new XML document. The query dispatch is not implemented yet at this stage due to limited sources.

The coordinate system of the IFC building model is different from the definition of geospatial coordinate system. Coordinate conversion is thus needed to transform the IFC model coordinates into the geospatial reference system, so that the IFC model can be geo-referenced.

In the merging process, one challenge is the coordinate transformation in order to georeference the IFC building information into the geo-information. The coordinate system of the IFC building model is different from the definition of geospatial coordinate system. Coordinate conversion was implemented to convert the IFC model coordinates into the geospatial coordinate reference system. Due to the lack of geo-referenced information in the current IFC building model, the building footprint matching process has to be undertaken by manually finding corresponding points between the IFC model and the geo-spatial data. A transform matrix was then calculated based on these registration points and the IFC building model coordinate conversion was then implemented automatically. The geo-spatial data of Jactin house area uses OSGB1936. The coordinate of IFC Jactin house building transformed into OSGB1936.

After the merging, a CityGML document is outputted as the integration result which include the Jactin House in LOD3 and surrounding building in LOD1.

\section{Client application}

The clients can assess the integrated results from browser and in their coding for any kinds of application. The client side sends the request based on the user's choices and communicates with the mediator to retrieve integrated information. The returned result is a CityGML document. A client can either be any standalone program that can parse this XML documents to present or visualise it. Or it can be any object embedded into a web browser under the system 
architecture. Figure 6 shows the integrated CityGML file in Aristoteles3D viewer ( http://131.220.71.208/index.php/Aristoteles).

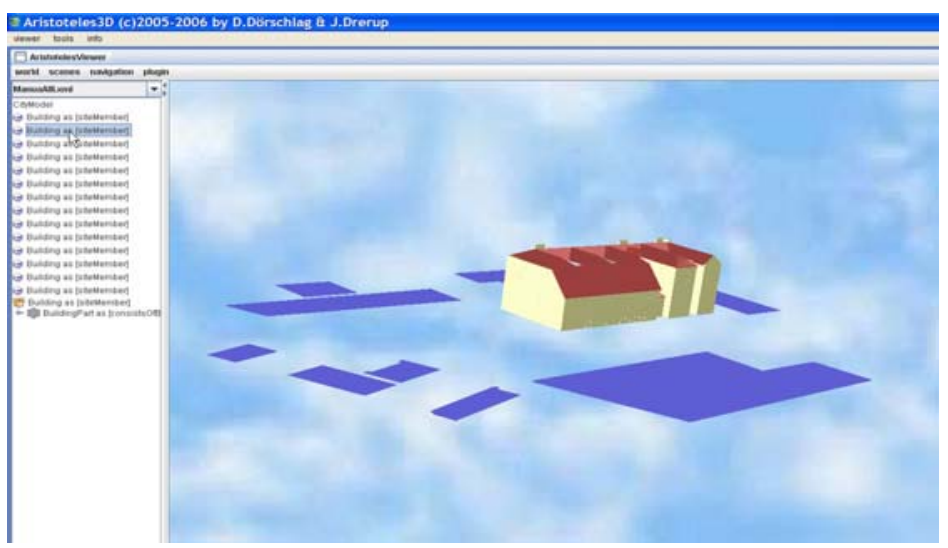

Figure 6: The integrated CityGML file displayed in Aristoteles3D viewer

\section{Summary}

In this paper, we introduced some research work being carried out by the authors. The Webbased information service framework is designed to support the sharing and integration of geospatial information, building information and other related information for urban planning application. Information sharing and integration are based on a loosely-coupled mediator system and OGC's Web Services architecture. BFS is defined to implement the retrieve of building information models on Web according to the OWS data service principle. The BFS can bring the rich-building information into the urban model. Further more, it can also act as a webbased information sharing and exchange service within the construction domain. The common model is based on GML/CityGML and IFC. The Mediator is designed to respond to the client request, retrieve the building features from a BFS and GIS Features from WFS, and send back the combined result. The first version of a prototype has been developed as proof of concept. Due to the limited resources, the prototype only are working on two types of datasets -- geodatabase and IFC building model.

In VEPS, this framework has been employed as the information service layer in the common architecture design. It is being used to share and integrate the building and geo-spatial information to support the interactive 3D visualisation application.

However, there are still some challenges that include the extension of common data model for more generic urban planning applications, geo-referencing IFC building models, and semantic integration by using ontology etc. The prototype is just an initial development effort as a proof of concept. Significant further work remains to be done in the future. 


\section{Acknowledgement}

The authors would like to thank EU's INTERREG IIIB North West Europe Programme and UK'S Office of Deputy Prime Minister (ODPM) for funding of VEPS Project.

\section{References}

Abel, D. J., Beng Chin Ooi, Kian-Lee Tan and Soon Huat Tan (1998). "Towards integrated geographical information processing." International journal of geographical information science vol. 12(no. 4): 353- 371.

Arayici, Y., Hamilton, A., Gamito, P., Albergaria, G. (2005). Using the 3D Laser Scanner Data Capture and Modelling to Visualise the Built Environment: Data Capture and Modelling. 9th International Conference of Information Visualisation, London.

Bishr, Y. (1998a). "Overcoming the semantic and other Barriers to GIS Interoperability." The international Journal of GIS 12(3).

Butenuth, M. a. C. H. (2003). Modelling The Integration of Heterogeneous Vector Data and Aerial Imagery.

Claramunt, C., B. Jiang, et al. (2000). "A New Framework for the Integration, Analysis and Visualisation of Urban Traffic Data Within GIS." Transportation Research Part C: Emerging Technologies, An International Journal: 167 - 184.

Dollner, J., Kolbe, Thomas H., Liecke, Falko, Sgouros, Takis, Teichmann, Karin (2006). The Virtual 3D City Model of Berlin - Managing, Integrating, and Communicating Complex Urban Information the 25th Urban Data Management Symposium UDMS 2006, Aalborg, Denmark.

Fileto, R. (2001). Issues on Interoperability and Integration of Heterogeneous Geographical Data. III Workshop Brasileiro de Geolnformatics, Rio de Janeiro, Braisl.

Garton, M. a. T., G. (2001). Data integration issues for a Farm Decision Support System. GIS Research in the UK 9th Annual Conference, Glamorgan.

Gröger, G., Kolbe, Thomas H., Czerwinski, Angela (2006). OpenGIS City Geography Markup Language (CityGML) Implementation Specification Version 0.3.0, Open Geospatial Consortium, Inc. $®$

Hamilton, A., H. Wang, et al. (2005). "Urban information model for city planning." ITcon 10(Special Issue From 3D to nD modelling): 55-67.

Holland, P. (1999). The Strategic Imperative of a Global Spatial Data Infrastructure. Cambridge Conference for National Mapping Organizations, Cambridge, UK.

IAI (2002). International Alliance for Interoperability. 2004.

INSPIRE(2004), Infrastructure for Spatial Information in Europe, http://www.ec-gis.org/inspire/. Accessed May 182005.

Jepson, W. H., R. S. Liggett, et al. (2001). An Integrated Environment for Urban Simulation. Planning Support Systems: Integrating Geographic Information Systems, Models, and Visualization Tools. R. K. Brail and R. E. Klosterman. Redlands, California, USA, ESRI Press: 387-404. 
Kolbe T. H. , G. G. G., Plümer Lutz (2005). CityGML - Interoperable Access to 3D City Models. International Symposium on Geoinformation for Disaster Management.

Mas, S. R., Wolfgang and Wang Fei (2006). Conception of a 3D geodata web service for the support of indoor navigation with gnss. Innovation in 3D geoinformation science lecture notes on geoinformation and cartgraphy. A. Z. Abudul-rahman, Sisi; Coors, Volker.: 307-316.

Murray, K. (2002). New Geo-information Framework for Great Britain. FIG XXII International Congress, Washington, D.C. USA.

Nedovic-Budica, Z., Feeneyb, M.-E.,F., Rajabifardb,A., Williamsonb, I. (2004). "Are SDIs serving the needs of local planning? Case study of Victoria, Australia and Illinois, USA." Computer, Environment and Urban Systems 28: 329-351.

NIBS (2005). Building Information Model (BIM). Washington, DC, National BIM Standard, Facilities Information Council (FIC) http://www.nibs.org/FIC/BIM82905/1 NIBS FMOC 82905 Minutes.pdf

OGC (2005). OpenGIS $₫$ Web Services Common Specification Version 1.0.0, Open Geospatial Consortium Inc.

OGC (2005a). Web Feature Service Implementation Specification Version 1.1.0, Open Geospatial Consortium Inc. Editor: Panagiotis A. Vretanos, 3 May 2005

OGC (2005b). OpenGIS $®$ Filter Encoding Implementation Specification Version 1.1.0, Open Geospatial Consortium Inc. Editor: Panagiotis A. Vretanos, 3 May 2005

Oosterom, P. v., Janttien Stoter, anbd Eric Janson (2005). Bridging the Worlds of CAD and GIS. Large-scale 3D Data Integration: Challenges and opportunities. S. Z. a. D. Prosperi. Boca Raton, Florida, CRC Press Taylor \&Francis Group: 9-38.

Raton, Florida, CRC Press Taylor \&Francis Group: 9-38.

Reed, C. (2005). Data Integration and Interoperability: ISO/OGC Standards for Geo-information. Large-scale 3D Data Integration: Challenges and opportunities. S. Z. a. D. Prosperi. Boca Raton, Florida, CRC Press Taylor \&Francis Group: 161-177.

Reinoso-Castillo, J., Silvescu, A., Caragea, D., Pathak, J., Honavar, V. (2003). Information extraction and integration from heterogeneous, distributed, autonomous information sources: a federated, query-centric approach. IEEE International Conference on Information Integration and Reuse, Las Vegas, Nevada.

Schilcher, M., Anderas Donaubauer (2005). OGC Specifications for Access to Distributed Geospatial Data. Photogrammetric Week 05', Wichmann Verlag, Heidelberg.

Sheth, A., Larson, L. (1990). "Federated database systems for managing distributed, heterogeneous and autonomous databases." ACM Computing Surveys 22: 183-236.

Stoimenov, L., Kajan, S. D. (2002). "Framwrok for Semantic GIS Interoperability." Ser. Math. Inform. 17.

Tatbul, N., O. Karpenko, et al. (2001) (2001). Data Integration Services. 
Teo Ai Lin, E., Cheng Tai Fatt (2006). "Building Smart - A Strategy for Implementing BIM Solution in Singapore." Synthesis Journal 2006 Section 5: 117-124.

Visser, U., Heiner Stuckenschmidt, Holger Wache, Thomas Vögele (2001). Using Environmental Information Efficiently: Sharing Data and Knowledge from Heterogeneous Sources. Environmental Information Systems in Industry and Public Administration. C. R. a. S. Patig, PA: Idea Group Publishing: 41-73.

Voisard, A., and Juergens, M. (1999). Geographic Information Extraction: Querying or Quarrying? Interoperating Geographic Information Systems. M. E. M. Goodchild, R. Fegeas and C. Kottman. New York, Kluwer Academic Publishers.

Wang, H., Hamilton, A. (2005). Data integration issues within nD Information Model for Urban Planning. , . 5th International Postgraduate Research Conference, Salford, UK, Blackwell Publishing.

Wiederhold, G. (1992). "Mediator in the architecture of furture information system." IEEE Computer 25(1): 38-49.

Wiederhold, G. (1994). Interoperation, Mediation and Ontologies. Sympoism. on Fifth Generation Computer Systems, Tokyo, Japan.

Yao, J., H. Tawfik, et al. (2003). Supporting Collaborative Urban Planning with GIS and Virtual Reality. GISRUK, City University, London.

Zhang, J., Javed, M., Shaheen, A., Gruenwald, L. (2000). Prototype for Wrapping and Visualizing Geo-Referenced Data in A Distributed Environment Using XML Technology. 8 th ACM Symposium on GIS 11/00 @ 2000, Washington, D.C., USA, ACM.

Zlatanova, S., A. A. Rahman, et al. (2002). 3D GIS: Current Status and Perspectives. the Joint Conference on Geo-spatial theory, Processing and Applications, Ottawa, Canada. 
